\title{
Perforating Rheumatoid Nodule Mimicking Malignant Soft-tissue Mass of the Forearm
}

Paul Ornetti ${ }^{-}$, MD, PhD, INSERM UMR1093-CAPS, Université Bourgogne Franche-Comté, and Plateforme d'investigation technologique, CIC-P, Rheumatology Department, François-Mitterrand Teaching Hospital; David Guillier, MD, PhD, Plastic and Reconstructive Department, François-Mitterrand Teaching Hospital; Geraldine Jeudy, MD, Dermatology Department, François-Mitterrand Teaching Hospital, Dijon, France. Address correspondence to Prof. P. Ornetti, Department of Rheumatology, 14 rue Gaffarel, François-Mitterrand Teaching Hospital, 21000 Dijon, France. Email: paul.ornetti@chu-dijon.fr. The authors report no financial disclosure or potential conflicts of interest or funding source. Ethics approval was waived according to the authors' institution. The patient's written informed consent was obtained to publish this manuscript.

Rheumatoid nodule ${ }^{1}$, one of the most characteristic manifestations of rheumatoid arthritis (RA), occurs in approximately $30 \%$ of patients, is generally associated with positive rheumatoid factor, smoking, and high disease activity ${ }^{2}$, and occurs in some patients with specific genotypes receiving methotrexate $(\mathrm{MTX})^{3}$.

An 83-year-old woman with a history of anticitrullinated protein antibody-positive RA treated by oral MTX, as well as gout, was hospitalized for a growing tumor of her right forearm. She had a 3-day history of intermittent fever. The clinical examination revealed a firm, painless subcutaneous mass with a $4 \mathrm{~cm}$ diameter budding and hemorrhagic ulceration, and an axillary lymphadenopathy. The radiograph showed a rounded softtissue thickening of the distal forearm and typical radiological features of RA in the right hand (Figure 1). Laboratory tests showed systemic inflammation with C-reactive protein levels of $94 \mathrm{mg} / \mathrm{L}$ (reference value $<5 \mathrm{mg} / \mathrm{L}$ ), a normal serum uric acid level (190 $\mu \mathrm{mol} / \mathrm{L}$, reference value $155-355 \mu \mathrm{mol} / \mathrm{L})$, and negative blood cultures. Ultrasonography and magnetic resonance imaging revealed a large encapsulated soft-tissue mass with peripheral inflammatory rim, whose appearance suggests a malignant tumor rather than a rheumatoid tenosynovitis or gouty tophi. The surgical biopsy exhibited nodular granulomatous tissue reaction pattern containing lymphocytes and

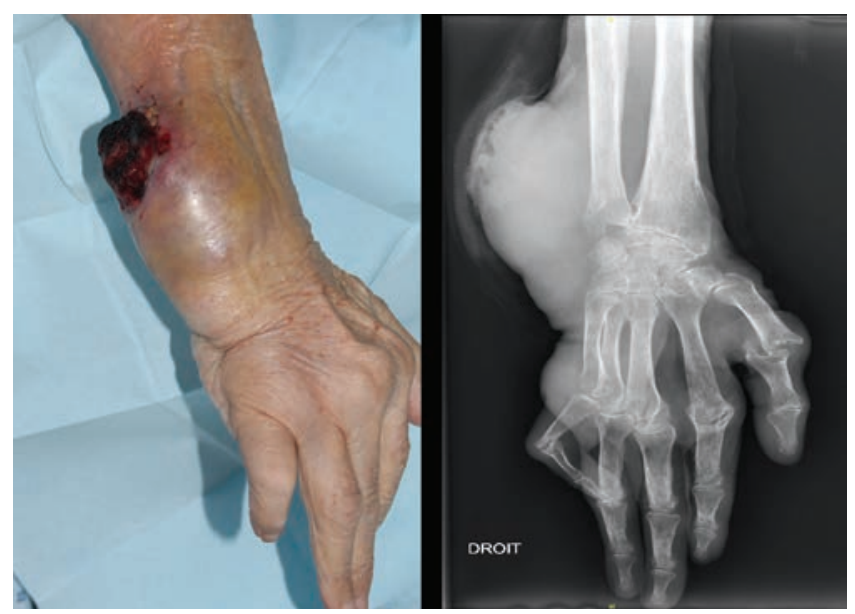

Figure 1. Right hand (radiograph and photograph): oblique view.

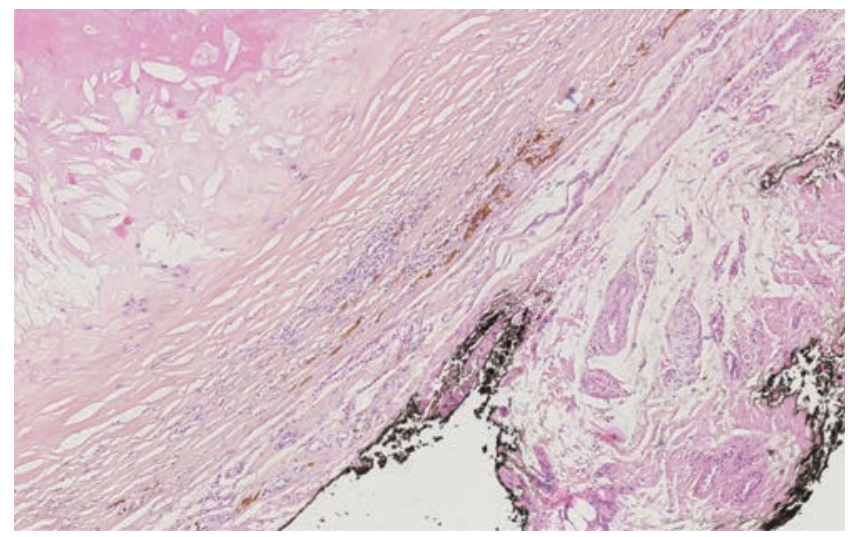

Figure 2. Histology of the lesion: rheumatoid nodule.

palisading epithelioid histiocytes, leading to the diagnosis of perforating rheumatoid nodule with subsequent local infection (Figure 2). The patient refused the complete surgical excision and was treated by oral antibiotics and dressings, which allowed the regression of clinical and biological inflammatory signs in a few days.

\section{ACKNOWLEDGMENT}

We thank Dr. M.H. Aubriot-Lorton (Department of Pathology, Dijon University Hospital) for assistance with the histopathologic report.

\section{REFERENCES}

1. Tilstra JS, Lienesch DW. Rheumatoid nodules. Dermatol Clin 2015;33:361-71.

2. Bang S, Kim Y, Jang K, Paik SS, Shin SJ. Clinicopathologic features of rheumatoid nodules: a retrospective analysis. Clin Rheumatol 2019;38:3041-8.

3. Soukup T, Dosedel M, Nekvindova J, Antonin Kubena A, Tacheci I, Duintjer Tebbens J, et al. The plausible association of MTHFR and ADORA2A polymorphisms with nodules in rheumatoid arthritis patients treated with methotrexate. Pharmacogenet Genomics 2017;27:43-50. 